\title{
Performance analysis of Poisson and exponential distribution queuing model in local area network
}

\begin{abstract}
Performance analysis of network queuing model is very important due to delay and latency that may be caused by using inappropriate model. It also impacts the utilization and throughput of the network. Many network queuing models have been proposed to be used in the network such as Poisson distribution model and Exponential distribution model. The first step in using a suitable queuing model is to know about the performance of that specific model. It means that a model with less delay and maximum utilization should be chosen to implement in the network. In this paper, performances of Poisson distribution and Exponential distribution methods in a queuing model are compared by OPNET Simulator. The behavior of these two queuing models is analyzed to find the best model in terms of queuing delay, utilization, throughput, and network load. Finally, a mathematical method is used to prove the result of simulation and find the model that performs well in the network. The results of this study show a better performance of Exponential distribute queuing model than Poisson distribute queuing model. In Exponential distribution queuing model not only does the network experience a higher throughput, but the network using Exponential distribution queuing model also maintains a less delay in the network.
\end{abstract}

Keyword: Component; Queuing model; Exponential distribution; Poisson distribution 